\title{
Impact of Speed Ripple on the Back-Emf Waveform of Permanent Magnet Synchronous Machines
}

\author{
Jeroen D. M. De Kooning, Jan Van de Vyver, Tine L. Vandoorn, Bart Meersman and Lieven Vandevelde
}

\begin{abstract}
Permanent Magnet Synchronous Machines (PMSMs) are frequently used in industry due to their high efficiency and favorable dynamic properties. Mechanical limitations and design considerations cause several harmonics in the flux and back-emf of these machines. The back-emf harmonics can be measured on the machine terminals if no stator current is present and the neutral point is accessible. The measured harmonics can then be included in a mathematical model of the machine. This measurement is often done for a constant speed. However, when a speed ripple is present, several new harmonics are introduced in the flux and back-emf. Although the existence of this phenomenon is intuitively clear, it has not yet been investigated in detail and no method exists to calculate these additional harmonics. Nevertheless, the impact of a speed ripple on the back-emf can become significant in some applications. Therefore, in this paper, a mathematical model is presented which allows to accurately calculate the additional back-emf harmonics in the presence of speed ripples. Also, it provides more insight in the interaction between speed ripples and harmonics. The mathematical model is extensively validated by means of simulations and experiments.
\end{abstract}

Index Terms-Permanent magnet machines, Brushless machines, Speed ripple, Back-emf harmonics, Vibrations, Modeling of electrical machines

\section{NOMENCLATURE}

$\delta_{E} \quad$ Rms of actual minus constant-speed back-emf

$\delta_{\Psi} \quad$ Rms of actual minus constant-speed flux

E Back-emf [V]

$E_{0} \quad$ Constant-speed back-emf [V]

$E_{v} \quad$ Ripple-induced Back-emf [V]

$J \quad$ Rotational inertia $\left[\mathrm{kgm}^{2}\right]$

$N_{h} \quad$ Harmonic order of the speed ripple

$N_{p} \quad$ Pole pair number

$\Omega \quad$ Mechanical speed [rad/s or $\mathrm{rpm}]$

$\Omega_{0} \quad$ Average speed [rad/s or $\mathrm{rpm}$ ]

PMSM Permanent Magnet Synchronous Machine

$\Psi \quad$ Flux of the permanent magnets [Wb]

$\Psi_{0} \quad$ Constant-speed flux [Wb]

$\Psi_{v} \quad$ Ripple-induced flux [Wb]

$T_{0} \quad$ Torque neglecting impact of speed ripple [Nm]

$T \quad$ Torque including impact of speed ripples [Nm]

$\theta_{r} \quad$ Mechanical shaft angle [rad]

J. D. M. De Kooning, J. Van de Vyver, T. L. Vandoorn, B. Meersman and L. Vandevelde are with the Electrical Energy Laboratory (EELAB), Department of Electrical Energy, Systems and Automation (EESA), Ghent University, Sint-Pietersnieuwstraat 41, B-9000 Ghent, Belgium, Telephone: +32 9264 34 22, Fax: +32 926435 82, e-mail: Jeroen.DeKooning@UGent.be

\section{INTRODUCTION}

Permanent Magnet Synchronous Machines (PMSMs) are popular in industry due to their high efficiency and favorable dynamic properties. They are manufactured in two forms, e.g., the brushless ac and the brushless dc machine. In brushless ac machines, the placement and shape of the magnets in combination with a distributed stator winding result in a sinusoidal waveform of the back-emf. They are used as a motor in high-efficiency drives or as a generator in renewable energy applications, such as wind energy and combined heat and power (CHP) systems [1], [2]. In brushless dc machines, a concentrated stator winding is used, theoretically resulting in a trapezoidal waveform of the back-emf. They are used in robotics, accurate positioning applications or servo drives [3].

Due to mechanical limitations and design considerations regarding the rotor magnets and the stator winding, the backemf in a PMSM does not perfectly match an ideal sinusoidal or trapezoidal waveform [4]. In general, the back-emf of a PMSM can be considered as a fundamental sinusoidal wave and harmonics. Also, subharmonics can be present in the back-emf, e.g., in PMSMs with a fractional slot winding [5], which is often used in axial flux machines. In a star-connected machine with accessible neutral, or in a machine with windings which are completely accessible, these harmonics can be identified during a constant-speed test without stator currents. From this, the harmonics of the flux coupled with the stator windings are easily calculated and can be integrated in a mathematical model of the machine.

In practice however, the speed is not always constant but can contain ripples [6]. For example, in the case of a wind turbine system, speed ripples can be caused by wind turbulence, tower shadow, wind shear or mechanical issues such as resonances [7], [8] and eccentricity. Several loads in industry generate pulsating torques or consume intermittent power, which causes vibrations, e.g., conveyor belt systems [9], piston pumps and compressors [10]. Speed ripples can also be caused by the PMSM itself, e.g., originated by torque ripples [11]-[13].

The speed ripples have an impact on the flux and back-emf waveform. The ripples can significantly change the harmonic spectrum and waveform of the back-emf when compared to the constant-speed situation. This effect is often overlooked or neglected, e.g., most mathematical models of the PMSM assume a constant speed. Neglecting this effect in vibrationsensitive situations could lead to unrealistic simulation results regarding dynamic behavior. For instance, the waveform of the back-emf can be used to calculate appropriate current harmonics to minimize torque ripple [14], [15]. When the 
back-emf waveform changes due to speed ripples, the calculated current harmonics are no longer correct and the torque ripple is not minimized. To achieve a constant torque, the impact of speed ripples should thus be included. Although it is intuitively clear that speed ripples can introduce additional harmonics in the flux and back-emf, this effect has not yet been investigated in detail in literature. Also, no methods exist in literature to calculate the frequency and amplitude of these additional harmonics for given speed ripples. Therefore, this paper presents a mathematical model in the frequency domain which allows to accurately calculate and predict these harmonics. The harmonics could also be calculated with a dynamical simulation model or Finite Element Methods. This does however not provide much insight and general conclusions cannot be made.

In this paper, the effect of speed ripples on the flux and the back-emf is discussed in detail. First, a mathematical model will be presented to calculate the harmonics in the presence of speed ripples, which is applicable for both brushless ac and dc machines. Second, a simplified analytical expression for the harmonics induced by speed ripples is derived, providing additional insight in these harmonics and their origin. Third, the model will be applied to two practical situations. In the first situation, a simple speed ripple is imposed on a PMSM which allows to validate the accuracy of the mathematical model. In the second situation, the speed ripples are caused by the PMSM itself, i.e., torque ripples. Finally, measurements are performed on a test-setup to compare measured and simulated waveforms.

\section{DEFINITIONS}

Four quantities are of interest in this paper, i.e., the rotational speed $\Omega$, the angular rotor position $\theta_{r}$, the flux $\Psi$ coupled with an arbitrary stator winding and the back-emf $E$. Fig. 1] gives a schematic two-pole equivalent representation of the PMSM, including the stator windings $a, b$ and $c$, the rotor axes $d, q$ and the mechanical rotor position $\theta_{r}$.

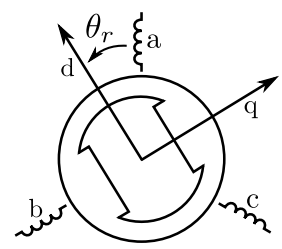

Fig. 1. Schematic representation of the PMSM

Each of the four quantities will be represented by a Fourier series. As the Fourier transformation is only applicable on periodic signals, a regime situation will be assumed. A finite time interval is chosen, equal to $\mu$ times one revolution of the rotor, i.e., $\mu 2 \pi / \Omega_{0}$, where $\Omega_{0}$ denotes the average speed and $\mu$ is a positive integer. The factor $\mu$ can be chosen depending on the application, e.g., $\mu=1$ if only 'conventional' back-emf harmonics are studied or $\mu>1$ if back-emf subharmonics or low-frequent speed ripples need to be included.

\section{A. Speed}

The starting point of this paper is the presence of speed ripples, caused by the load or by the PMSM itself. The mechanical speed $\Omega(t)$ can be written as the sum of an average speed $\Omega_{0}$ and a Fourier series:

$$
\Omega(t)=\Omega_{0}+\sum_{n=-\infty}^{+\infty} \hat{\Omega}(n) \cdot e^{j n \Omega_{0} t / \mu}
$$

The complex coefficients $\hat{\Omega}(n)$ are calculated with the Fourier transformation:

$$
\hat{\Omega}(n)=\frac{\Omega_{0}}{\mu 2 \pi} \int_{0}^{\mu \frac{2 \pi}{\Omega_{0}}} \Omega(t) \cdot e^{-j n \Omega_{0} t / \mu} d t
$$

\section{B. Angular rotor position}

The angular rotor position or mechanical shaft angle $\theta_{r}(t)$ can be calculated from $\Omega(t)$ by integrating $(1)$ :

$$
\theta_{r}(t)=\int_{0}^{t} \Omega(t) d t=\Omega_{0} t+\sum_{n=-\infty}^{+\infty} \mu \frac{\hat{\Omega}(n)}{j n \Omega_{0}} \cdot e^{j n \Omega_{0} t / \mu}
$$

The second term is a series of harmonics caused by the speed ripples. Their amplitudes are clearly attenuated due to the division of $n$. However, this does not mean they can be neglected, as will become clear further in this paper.

\section{Flux}

The back-emf of a PMSM is induced by the flux coupled with the stator winding. This total flux is the superposition of two components, i.e., the flux of the permanent magnets of the rotor and the flux caused by the stator windings themselves. The first component is the main flux component while the second component is a smaller additional effect due to armature reaction. The permanent magnet flux contains several spatial harmonics in the air-gap of the machine. A large part of this flux is coupled with the stator-winding and is capable of inducing a back-emf. The winding factors of the stator winding, defined by its design, determine how strong the different harmonics are coupled with the stator. This results in a stator-coupled flux waveform containing harmonics. In this paper, only the dominant flux component caused by the permanent magnets is considered. The impact of speed ripples on the armature reaction flux is not considered here, as it is outside the scope of this paper. Since this armature reaction flux can be seen as decoupled from the permanent magnet flux, this does not decrease the validity of the model developed in this paper.

The waveform of the flux $\Psi$, can be written as the following Fourier series in terms of $\theta_{r}$ :

$$
\Psi\left(\theta_{r}(t)\right)=\sum_{k=-\infty}^{+\infty} \hat{\Psi}(k) \cdot e^{j k \theta_{r}(t) / \mu}
$$

In contrast to the Fourier series of $\Omega(t)$ and $\theta_{r}(t)$, this is not a Fourier series in time but in space, i.e., the rotor angle $\theta_{r}$. The coefficients $\hat{\Psi}(k)$ are therefore space harmonics and 
not time harmonics. The coefficients $\hat{\Psi}(k)$ are calculated from the flux $\Psi\left(\theta_{r}\right)$ by using the Fourier transformation:

$$
\hat{\Psi}(k)=\frac{1}{\mu 2 \pi} \int_{0}^{\mu 2 \pi} \Psi\left(\theta_{r}\right) \cdot e^{-j k \theta_{r} / \mu} d \theta_{r}
$$

In agreement with the time period $\mu 2 \pi / \Omega_{0}$, a period of $\mu 2 \pi$ is used here in the transformation since it also corresponds to $\mu$ times one revolution of the shaft. The consequence of this choice is that conventional harmonics have an order $k$ equal to a multiple of $\pm \mu N_{p}$. The fundamental component results in two frequency components, i.e., one with an order $\mu N_{p}$ and one with an order $-\mu N_{p}$. When $\mu$ is chosen large enough, also subharmonics can be included in the analysis. For instance, flux subharmonics are often present in PMSMs with fractional slot windings [5].

It is important to note that the Fourier series of the flux given in (4) depends on $\theta_{r}$. This angle itself has a Fourier series given by (3). In other words, the Fourier series (4) contains a second Fourier series (3). This complicates the mathematical model but will be treated appropriately in $\$$ III

\section{Back-emf}

The back-emf $E$ induced by the flux is calculated by:

$$
E=\frac{d \Psi}{d t}
$$

The derivative causes higher-order flux time harmonics to be magnified in the back-emf. This shows the importance of these flux harmonics, even if their amplitude seems negligible.

\section{E. Constant-speed situation}

When no speed ripples are present, the shaft angle becomes linear, i.e., $\Omega_{0} t$. The flux $\Psi$ and back-emf $E$ simplify to:

$$
\begin{aligned}
& \Psi_{0}(t)=\sum_{k=-\infty}^{+\infty} \hat{\Psi}(k) \cdot e^{j k \Omega_{0} t / \mu} \\
& E_{0}(t)=\sum_{k=-\infty}^{+\infty} \frac{j k \Omega_{0}}{\mu} \cdot \hat{\Psi}(k) \cdot e^{j k \Omega_{0} t / \mu}
\end{aligned}
$$

Equations (7) and (8) show that the flux harmonics of (4), which are space harmonics, are directly converted to time harmonics in the flux $\Psi_{0}(t)$. Therefore, the fluxes $\Psi\left(\theta_{r}\right)$ and $\Psi_{0}(t)$ have exactly the same waveform.

In [16], the back-emf waveform of a PMSM was measured and the dominant harmonics were given, i.e., a third harmonic of $22.45 \%$, a fifth harmonic of $5.43 \%$ and a seventh harmonic of $0.87 \%$. This waveform has been used here to construct Fig. 2, which shows the flux and back-emf as calculated by (7) and (8). The speed $\Omega$ equals $750 \mathrm{rpm}$, i.e., the synchronous speed for a $50 \mathrm{~Hz}$ machine with a pole pair number $N_{p}$ of four. The back-emf waveform is typical for a brushless dc machine.

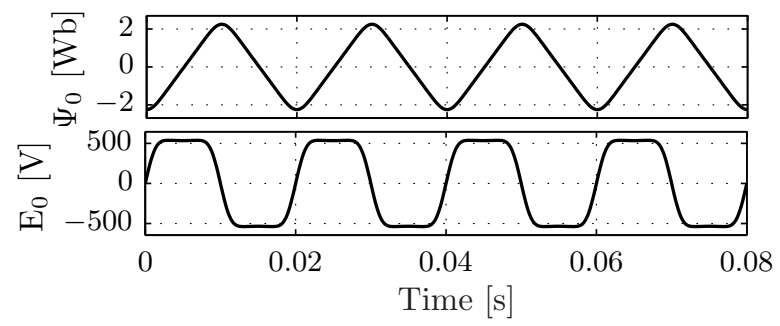

Fig. 2. Constant speed situation: flux $\Psi_{0}$ and back-emf $E_{0}$

\section{HARMONIC MODEL INCLUDING SPEED RIPPLES}

In this section, the definitions of $\Omega, \theta_{r}, \Psi$ and $E$ are applied to a more general situation where speed ripples are present. This leads to a harmonic model of the flux and back-emf, including an analytical expression for the harmonics caused by the speed ripples.

The combination of (3) and (4) gives the flux $\Psi(t)$ in the presence of speed ripples:

$$
\begin{aligned}
\Psi(t)= & \sum_{k=-\infty}^{+\infty} \hat{\Psi}(k) \cdot e^{j k \Omega_{0} t / \mu} \\
& \exp \left(j k \sum_{n=-\infty}^{+\infty} \frac{\hat{\Omega}(n)}{j n \Omega_{0}} \cdot e^{j n \Omega_{0} t / \mu}\right)
\end{aligned}
$$

This equation is not straightforward, since it contains a complex exponential function, which in turn contains a complex exponential function of time. However, as long as the ripples $\hat{\Omega}(n)$ are limited, the argument of the second exponential function in this equation is small enough to apply a first-order Taylor approximation, i.e., $\exp (x) \simeq 1+x$. This considerably simplifies the equation. The accuracy of this approximation will be verified further in this paper. The flux can now be written as follows:

$$
\begin{aligned}
& \Psi(t)=\sum_{k=-\infty}^{+\infty} \hat{\Psi}(k) \cdot e^{j k \Omega_{0} t / \mu} \\
& \left(\sum_{k=-\infty}^{+\infty} k \hat{\Psi}(k) \cdot e^{j k \Omega_{0} t / \mu}\right) \cdot\left(\sum_{n=-\infty}^{+\infty} \frac{\hat{\Omega}(n)}{n \Omega_{0}} \cdot e^{j n \Omega_{0} t / \mu}\right)
\end{aligned}
$$

The first term is equal to the constant speed flux $\Psi_{0}$. The second term is the product of two Fourier series with the same periodicity $2 \pi \mu / \Omega_{0}$. This second term can therefore be rewritten as a single Fourier series and will be called the ripple-induced flux $\Psi_{v}$, which results in:

$$
\begin{aligned}
& \Psi(t)=\sum_{k=-\infty}^{+\infty} \hat{\Psi}(k) \cdot e^{j k \Omega_{0} t / \mu}+\sum_{v=-\infty}^{+\infty} \hat{\Psi}_{v}(v) \cdot e^{j v \Omega_{0} t / \mu} \\
& =\quad \Psi_{0}(t)+\Psi_{v}(t)
\end{aligned}
$$

The Fourier coefficients $\hat{\Psi}_{v}(v)$ of the ripple-induced flux are calculated with a discrete convolution or Cauchy product: 


$$
\begin{aligned}
\hat{\Psi}_{v}(v) & =(k \hat{\Psi}(k)) *\left(\frac{\hat{\Omega}(n)}{n \Omega_{0}}\right) \\
& =\sum_{s=-\infty}^{+\infty} \frac{s}{v-s} \cdot \hat{\Psi}(s) \cdot \frac{\hat{\Omega}(v-s)}{\Omega_{0}}
\end{aligned}
$$

The superposition of the flux as the sum of the classical constant-speed flux and an additional ripple-induced flux is an elegant and convenient result. More specifically, the combination of a constant speed flux harmonic with order $k$ and a speed ripple with order $n$ causes one flux harmonic with order $k+n$ and one with order $k-n$.

The back-emf $E$ can also be split up into two parts, i.e., the classical constant-speed term $E_{0}$ and a ripple-induced term $E_{v}$ :

$$
\begin{aligned}
& E(t)=\sum_{k=-\infty}^{+\infty} \frac{j k \Omega_{0}}{\mu} \hat{\Psi}(k) e^{j \frac{k \Omega_{0}}{\mu} t}+\sum_{v=-\infty}^{+\infty} \frac{j v \Omega_{0}}{\mu} \hat{\Psi}_{v}(v) e^{j \frac{v \Omega_{0}}{\mu} t} \\
& =E_{0}(t)+E_{v}(t)
\end{aligned}
$$

The previously drawn conclusions for the flux induced by speed ripples are equally valid for the back-emf induced by speed ripples. The only approximation made in the derivation of the model is the first order Taylor series expansion.

\section{APPLICATION OF THE MODEL}

The model of the previous section has been programmed in Matlab and two situations are simulated. In the first situation, a speed ripple is imposed on a PMSM. In the second situation, a PMSM with a torque ripple is simulated. For both situations, the parameter $\mu$ is set at 1 such that subharmonics are not included.

\section{A. Imposed speed ripple}

The following speed is now imposed on the PMSM:

$$
\begin{aligned}
\Omega(t) & =\Omega_{0}+\frac{\hat{\Omega}\left(N_{h}\right)}{2} \cdot e^{j N_{h} \Omega_{0} t}+\frac{\hat{\Omega}\left(N_{h}\right)^{*}}{2} \cdot e^{-j N_{h} \Omega_{0} t} \\
& =\Omega_{0}+\hat{\Omega}\left(N_{h}\right) \cdot \cos \left(N_{h} \Omega_{0} t\right)
\end{aligned}
$$

As in the constant-speed simulation, the speed $\Omega_{0}$ is set at $750 \mathrm{rpm}$ and the pole pair number $N_{p}$ is set at 4 . The frequency of the ripple is expressed by the order $N_{h}$, which has a value of 8 . Four different simulations are performed, each with a different real-valued ripple amplitude $\hat{\Omega}(8)$, i.e., $10 \%, 30 \%$, $60 \%$ and $90 \%$ of $\Omega_{0}$ respectively. Fig. 3 shows the resulting flux waveforms.

For each simulation, three flux waveforms are shown. The dotted line represents the constant speed flux $\Psi_{0}$, calculated by (7) and shown in Fig. 2 as well. The full line shows the real flux $\Psi$ given by (9). The dashed line shows the flux $\Psi_{0}+\Psi_{v}$, which is the flux approximated by the model, given by 11 and (12). Fig. 4 shows the back-emf waveforms. Again, the dotted line shows the constant speed back-emf $E_{0}$ given by

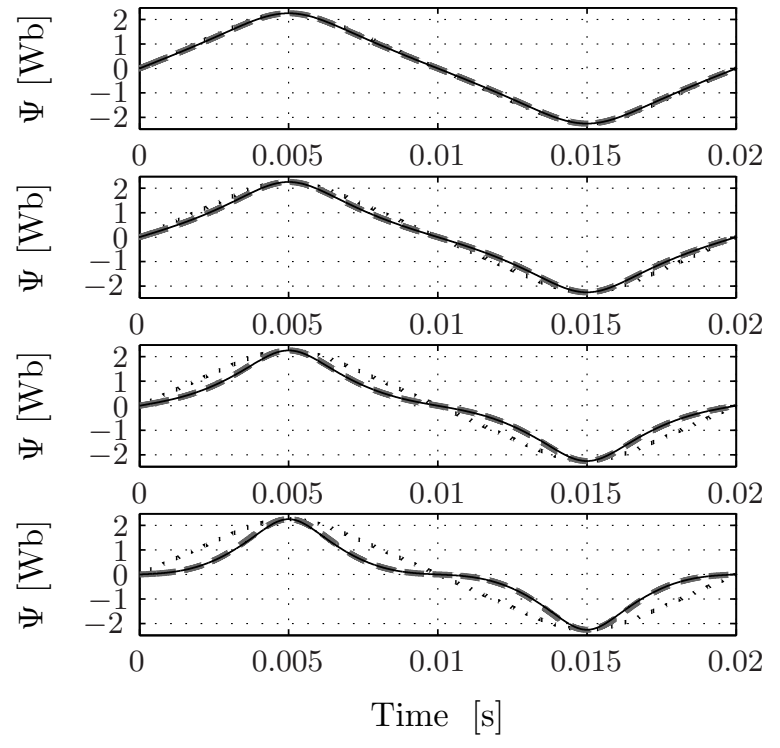

Fig. 3. Flux waveforms for an imposed speed ripple: (a) $\hat{\Omega}(8)=0.10 \Omega_{0}$, (b) $\hat{\Omega}(8)=0.30 \Omega_{0}$, (c) $\hat{\Omega}(8)=0.60 \Omega_{0}$, (d) $\hat{\Omega}(8)=0.90 \Omega_{0}$, $\ldots=$ constant-speed waveform, $-=$ real waveform, - - = simplified model

(8), the full line shows the real back-emf $E$ given by $(6)$ and the dashed line shows the approximated back-emf $E_{0}+E_{v}$ given by 13 .
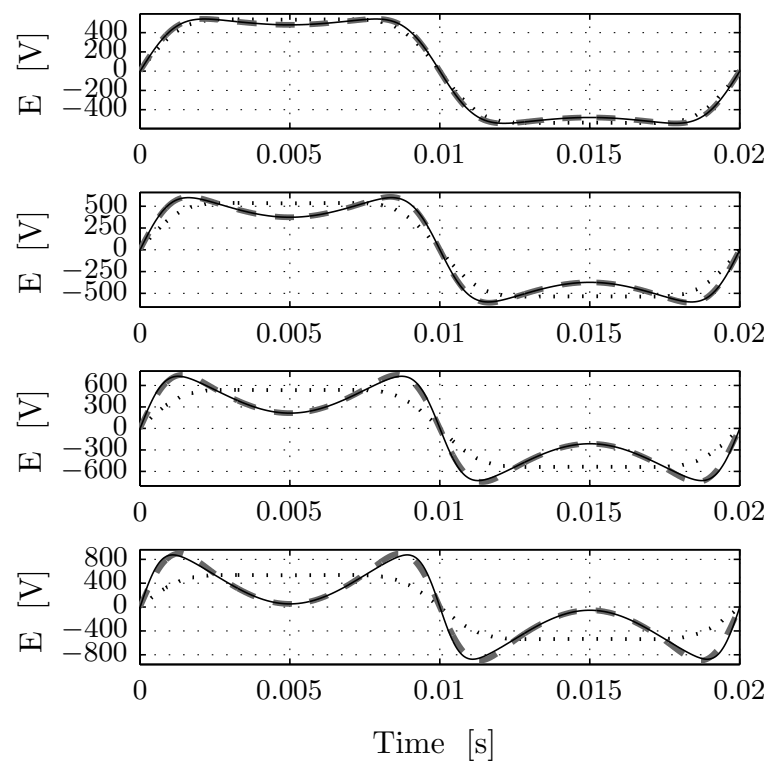

Fig. 4. Back-emf waveforms for an imposed speed ripple: (a) $\hat{\Omega}(8)=0.10 \Omega_{0}$, (b) $\hat{\Omega}(8)=0.30 \Omega_{0}$, (c) $\hat{\Omega}(8)=0.60 \Omega_{0}$, (d) $\hat{\Omega}(8)=0.90 \Omega_{0}$, .. = constant-speed waveform, - = real waveform, - - = simplified model

In situation (a), where the speed ripple is only $10 \%$, the three flux waveforms are similar since the flux components caused by the speed ripple are small. However, in the backemf waveforms, the effect of the ripple is visible, since $E$ and $E_{0}+E_{v}$ differ from $E_{0}$. The real back-emf $E$ and the approximated back-emf $E_{0}+E_{v}$ have a perfect match, so the model is valid in this case. In situation (b), where the ripple is $30 \%$, a small difference between the real flux $\Psi$ and the constant speed flux $\Psi_{0}$ can be observed. However, the 
difference between $E$ and $E_{0}$ has become significant. Still, the approximated waveforms match the real waveforms accurately enough such that the model remains valid. In situation (c), where a large ripple of $60 \%$ is present, there is a considerable difference between $\Psi$ and $\Psi_{0}$. In the back-emf waveforms, the real waveform $E$ and the constant speed waveform $E_{0}$ have a large deviation since the ripple-induced back-emf $E_{v}$ has an important influence. Despite the large speed ripple, the approximated waveforms are still close to the real waveforms. In situation (d), an extreme speed ripple of $90 \%$ causes a large ripple-induced flux $\Psi_{v}$ and back-emf $E_{v}$. Although a speed ripple of $90 \%$ has no practical relevance, it remains interesting to simulate this case in order to validate the mathematical accuracy of the model. In the back-emf, a small difference is noticeable between the model and the real waveform, which could be expected since the Taylor approximation is only valid for small ripples. However, the model still corresponds very well with the real waveform. Thus, the approximation made for the model remains valid even for extreme speed ripples.

The ripple in the speed $\Omega$ has an order $k$ of 8 . The constantspeed flux $\Psi_{0}$ includes components with an order $n$ of 4,12 , 20 and 28. The ripple flux $\Psi_{v}$ includes components with an order $v$ of 4,12, 20, 28 and 36. This confirms that the orders $v$ result from combining the orders $k$ and $n$ as $k \pm n$.

The previous simulation has shown how a speed ripple can influence the waveforms of the flux and the back-emf. It has also shown the validity of the model. Only for extreme ripples, the model is no longer valid. The simulation however has only shown the particular case of an order $N_{h}$ equal to 8. Therefore, these simulations will now be repeated for other orders and amplitudes, which allows to make a more general conclusion concerning the accuracy. The accuracy will be quantified by calculating the root mean square of the difference between the actual waveform and the constant speed waveform:

$$
\begin{aligned}
& \delta_{\Psi}=\frac{1}{\Psi_{0, \mathrm{rms}}} \cdot \sqrt{\frac{1}{T} \int_{0}^{T}\left(\Psi-\Psi_{0}\right)^{2} d t} \\
& \delta_{E}=\frac{1}{E_{0, \mathrm{rms}}} \cdot \sqrt{\frac{1}{T} \int_{0}^{T}\left(E-E_{0}\right)^{2} d t}
\end{aligned}
$$

Due to $(11)$ and $(13)$, these quantities equal the rms value of the flux and back-emf components caused by the speed ripples. They are divided by the rms values $\Psi_{0, \text { rms }}$ and $E_{0 \text {,rms }}$, such that $\delta_{\Psi}$ and $\delta_{E}$ are relative quantities. A high $\delta$ means that the ripple causes a large deviation between the real waveform and the constant-speed waveform.

Fig. 5 shows both $\delta_{\Psi}$ and $\delta_{E}$ in function of the ripple amplitude $\hat{\Omega}_{h}$ for different orders $N_{h}$ as defined in 14 . This calculation shows that the accuracy of the model decreases for large ripple amplitudes $\hat{\Omega}_{h}$, which is expected since the argument of the exponential function in 9 ) becomes large such that the Taylor approximation loses accuracy. Nevertheless, the calculation shows that the model has a high overall accuracy.

Fig. 6 shows both $\delta_{\Psi}$ and $\delta_{E}$ in function of discrete values of the order $N_{h}$ for different ripple amplitudes $\hat{\Omega}_{h}$. The values calculated from the model have a perfect agreement with the real values if the order $N_{h}$ is sufficiently large. For small
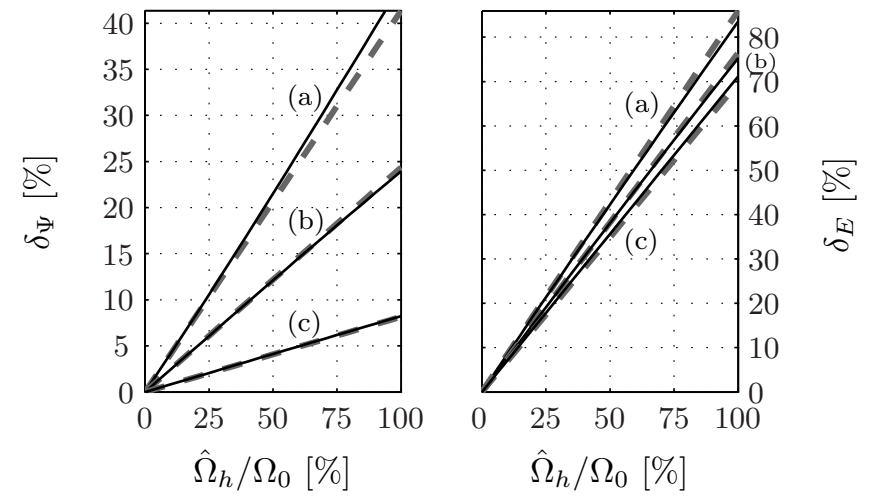

Fig. 5. $\delta_{\Psi}$ and $\delta_{E}$ in function of the ripple amplitude $\hat{\Omega}_{h}$ :

(a) $N_{h}=7$, (b) $N_{h}=12$, (c) $N_{h}=35,-=$ Calculated from real $\Psi$ and $E$, - - = Calculated from approximated $\Psi$ and $E$

values of $N_{h}$, the accuracy is reduced, which can be explained by the limited validity of the Taylor approximation.
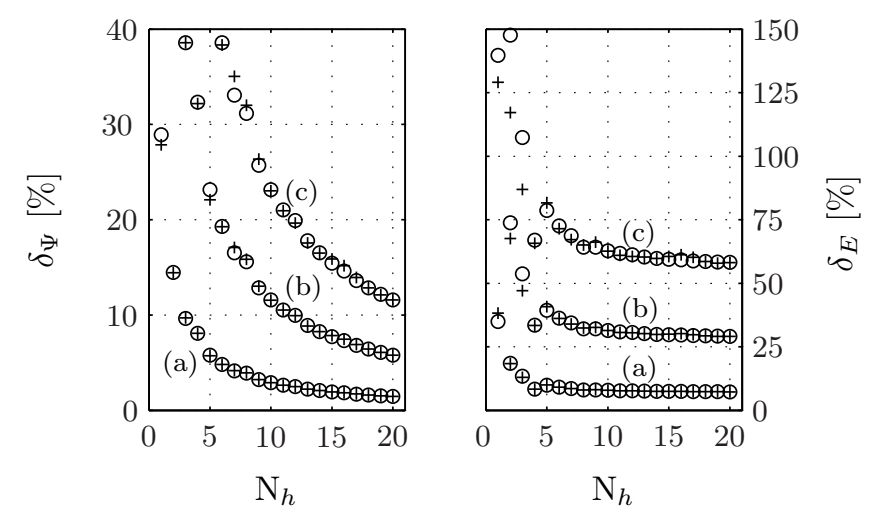

Fig. 6. $\delta_{\Psi}$ and $\delta_{E}$ in function of the order $N_{h}$ :

(a) $\hat{\Omega}_{h}=10 \%$, (b) $\hat{\Omega}_{h}=40 \%$, (c) $\hat{\Omega}_{h}=80 \%,+=$ Calculated from real $\Psi$ and $E$, $\circ=$ Calculated from approximated $\Psi$ and $E$

It is concluded that the simplified model has a high accuracy when compared to the real waveforms. Also, it offers a significant improvement when compared to the constant-speed model and is thus capable of accurately predicting the backemf of a PMSM in the presence of speed ripples.

\section{B. Torque ripple}

The concept of the ripple-induced flux and ripple-induced back-emf will now be applied on a PMSM connected to a constant-torque load. This load ensures that the speed ripples are only caused by the PMSM itself. Torque ripples are often present in PMSMs and have been widely discussed in literature, since they can have a negative effect on certain loads [17]-[19]. Torque ripples arise for example when sinusoidal currents are injected into a brushless motor with harmonics in the back-emf. The back-emf harmonics cause ripples in the mechanical power and, thus, also in the torque. The torque of the PMSM will be calculated from the speed, the back-emf and current waveforms of the three phases by using:

$$
T=\frac{E_{a} I_{a}+E_{b} I_{b}+E_{c} I_{c}}{\Omega}
$$


A sinusoidal current with an amplitude of $10 \mathrm{~A}$, in phase with the fundamental component of the back-emf, will be imposed on the machine. This can be achieved in practice with a current-controlled converter and an encoder as a position sensor, resulting in a field-oriented situation [20]. The current waveform used here is representative for a practical vectorcontrolled drive, as it also contains the switching ripple. This current waveform was obtained by simulating the vectorcontrolled drive with a switching frequency of $5 \mathrm{kHz}$ connected to the PMSM with the machine parameters of [16]. The effect of this current ripple on the torque, speed and subsequently back-emf waveform will also be discussed here.

The mean speed $\Omega_{0}$ is set at $750 \mathrm{rpm}$ and $N_{p}$ equals 4 . It is assumed that the torque of the constant-torque load equals the average torque $\bar{T}$ of the PMSM, which is the case in steadystate. The speed ripples can be calculated from the mechanical equation:

$$
\Omega=\Omega_{0}+\frac{1}{J} \int(T-\bar{T}) d t
$$

The goal of the simulation is to show that the torque ripple is influenced by including the impact of ripples on the backemf. Therefore, the torque ripple with and without the rippleinduced back-emf will be calculated and compared. For this, the inertia $J$ is set at a low value of $1 \mathrm{gm}^{2}$ to make the effect clearly visible.

Fig. 7 shows the resulting speed, current waveforms, backemf and torque. The current waveforms are sinusoidal with a current ripple. The combination of these current waveforms with the back-emf harmonics, clearly causes torque ripples. The torque waveforms contain a nearly sinusoidal low-frequent ripple component, caused by the dominant third harmonic in the flux and back-emf, and a high-frequent ripple caused by the switching ripple. The low-frequent torque ripple causes a speed ripple which is also nearly sinusoidal. On the other hand, the high-frequent torque ripple has a limited impact on the speed as it is more effectively filtered by the inertia.

The constant-speed back-emf $E_{0}$ is shown as a grey dashed line while the real back-emf $E$ is shown as a black full line. $E$ diverts from $E_{0}$ due to the speed ripples. To further clarify this, the waveform $E_{v}$ (calculated as $E-E_{0}$ ) is also shown. For the first torque waveform $T_{0}$, the constant-speed backemf $E_{0}$ and the real speed $\Omega$ were used in $(17)$. The torque $T_{0}$ can therefore be interpreted as the result of the classical approach, where the impact of speed ripples on the back-emf is neglected. For the second torque waveform $T$, the real backemf $E$ and the real speed $\Omega$ were used. The torque $T$ can therefore be interpreted as the real torque which would occur in practice. $T$ clearly diverts from $T_{0}$ due to the speed ripples.

Both $T_{0}$ and $T$ have the same fundamental frequency. The amplitude and phase are however changed by taking into account the effect of the speed ripples. The fundamental torque ripples of $T_{0}$ and $T$ have an amplitude of $7.38 \mathrm{Nm}$ and $4.46 \mathrm{Nm}$ respectively. Fig. 8 shows a Fourier amplitude spectrum of $T$ and $T_{0}$ on a logarithmic scale. These data were calculated with a Fast Fourier Transform algorithm applied on the waveforms of $T_{0}$ and $T$ with a high sampling frequency of
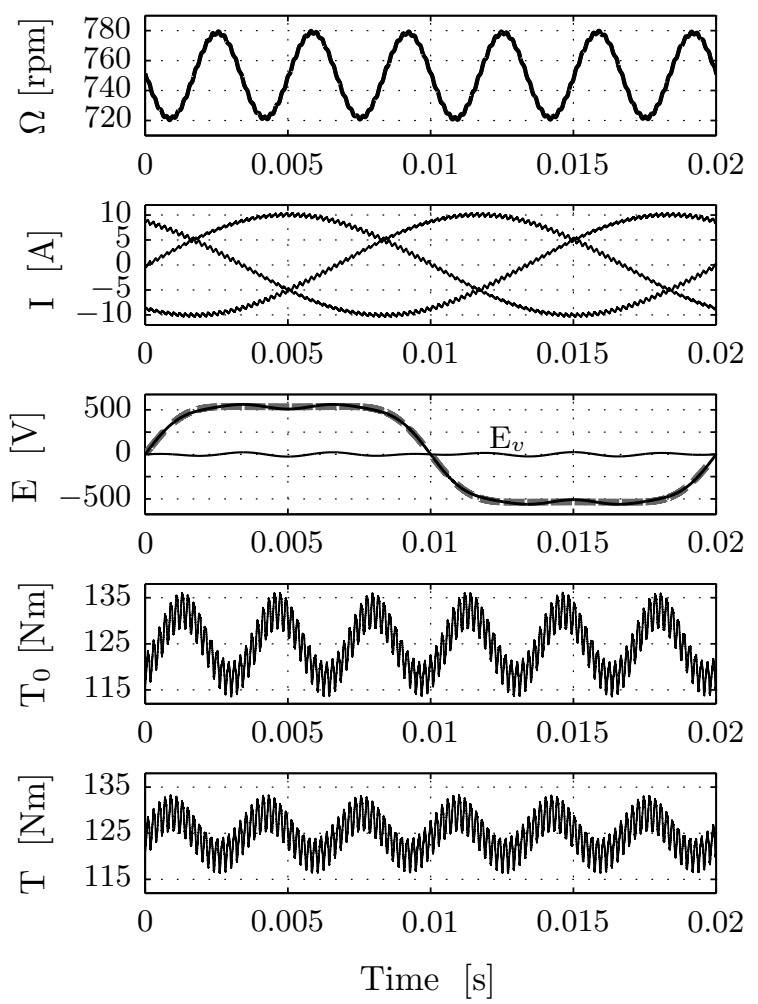

Fig. 7. PMSM with torque ripple: speed $\Omega$, currents $I$, back-emf $E$, torques $T_{0}$ and $T ;--=$ neglecting speed ripples, $-=$ including speed ripples

$50 \mathrm{MHz}$. Both $T_{0}$ and $T$ have two dominant components, i.e., 24 and 48, which is expected for a three-phase machine with 4 pole pairs and a third harmonic in the back-emf since the order of the torque ripples can be calculated as $3(k \pm 1) N_{p}$. Both dominant components are affected by the speed ripples. The other components have a smaller amplitude, i.e., mostly below $10^{-2} \mathrm{Nm}$. Nevertheless, these components are also slightly affected by the speed ripples.
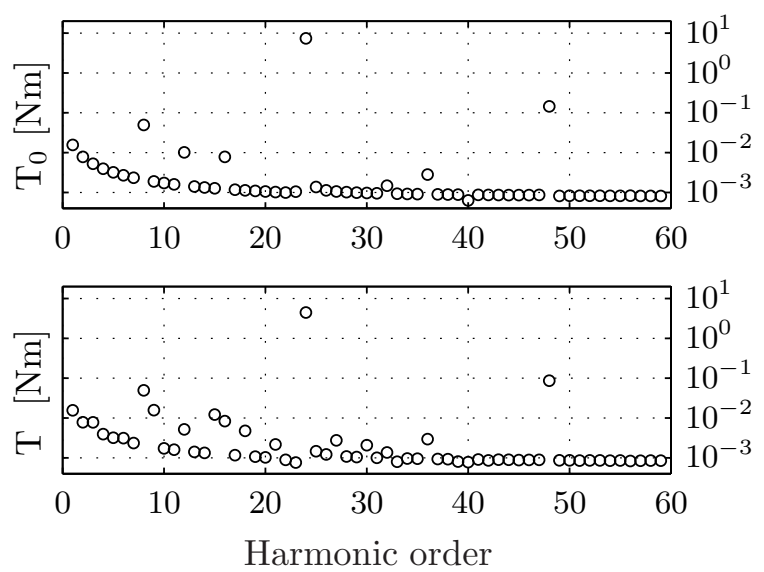

Fig. 8. Fourier amplitude spectrum of $T_{0}$ and $T$ on a logarithmic scale

\section{EXPERIMENTAL RESULTS}

The impact of speed ripples on the back-emf of a PMSM has been measured on a test-setup, shown schematically in 
Fig. 9 Measurements have been performed with and without an electrical load connected to the machine terminals.

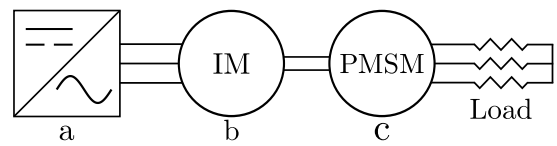

Fig. 9. Overview of the test-setup

The setup consists of an inverter (a) connected to an induction motor (b), which drives a PMSM (c). The inverter is a $2.2 \mathrm{~kW}$ Danfoss AutomationDrive FC302, which is programmed to control the speed of the induction motor. The motor is a standard $2.2 \mathrm{~kW}$ KEB machine with 2 pole pairs. The set-point for the speed is created with a Digital Signal Processor and can be varied in time to create speed ripples. The PMSM is a Mecc Alte Eogen 60/16 wind turbine generator. The parameters of this PMSM are given in Table I] The speed is measured with an encoder with 8096 pulses per revolution. From the PMSM's point of view, the motor and inverter emulate a load with a strongly pulsating torque. These pulsations will be set rather strong in the experiments to clearly see the effect on the back-emf waveform.

TABLE I

PARAmeters PMSM: Mecc Alte Eogen 60/16

\begin{tabular}{|c|c|c|c|c|c|c|}
\hline$P_{m}$ & $N_{p}$ & $\Omega_{\text {nom }}$ & $V_{\text {nom }}$ & Weight & $R_{s}$ & $L_{s}$ \\
\hline $1 \mathrm{~kW}$ & 8 & $415 \mathrm{rpm}$ & $400 \mathrm{~V}$ & $28 \mathrm{~kg}$ & $13.47 \Omega$ & $120 \mathrm{mH}$ \\
\hline
\end{tabular}

In a first measurement, the speed setpoint is kept constant to measure the constant-speed back-emf waveform $E_{0}$. This measurement is repeated for different speeds, i.e., 92, 177, 303 and $434 \mathrm{rpm}$. The measured data are cut off at the 9th harmonic. Finally, from these measurements an averaged waveform $E_{0}$ can be calculated, which is shown in Fig. 10

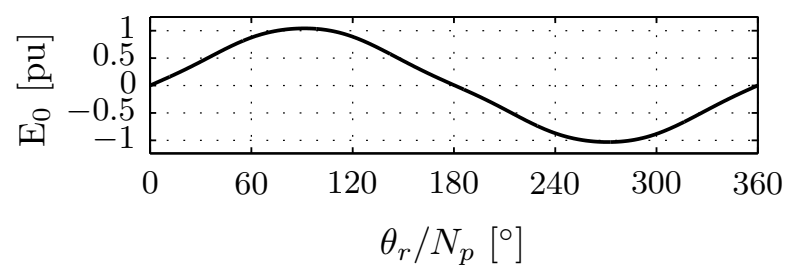

Fig. 10. Measured constant speed back-emf waveform $E_{0}$

The measured waveform is scaled such that the fundamental component has a magnitude of $1 \mathrm{pu}$. The waveform contains a third harmonic of $5.2 \%$ and a fifth harmonic of $1.4 \%$. The other components are negligible. Instead of the previouslyused waveform of [16], this measured waveform is now included in the simulation model.

\section{A. No-load measurement}

Now that the constant-speed waveform $E_{0}$ is known, a measurement with a speed ripple is performed. No electrical load is connected to the PMSM, such that the back-emf waveform can be measured directly on the machine terminals. The setpoint of the speed controller in the inverter is varied sinusoidally around a constant average value. Fig. 11 shows the result of this measurement, together with simulated data.
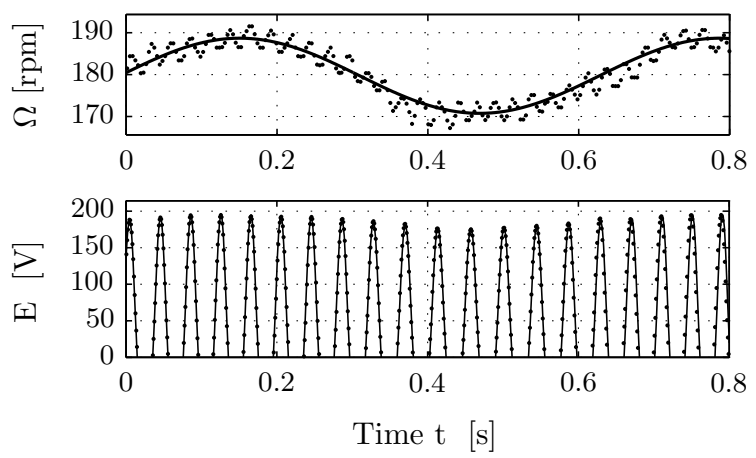

Fig. 11. Measured speed ripple: $\Omega(t)$ and $E(t),-=$ simulated, $\ldots=$ measured.

The speed of the PMSM was measured with a sampling frequency of $300 \mathrm{~Hz}$ and has an average value $\Omega_{0}$ of $180 \mathrm{rpm}$ and several harmonics. The dominant component (full line) has a frequency of $1.57 \mathrm{~Hz}$ and an amplitude of $9 \mathrm{rpm}$ ( $5 \%$ of $\Omega_{0}$ ). This dominant component was imported in the simulation model to simulate the back-emf $E$. Since the ripple is subharmonic, the parameter $\mu$ was set at 10 . For the backemf, only the positive side of the waveform is shown to clearly show the amplitude variation. There is a clear correspondence between the simulated back-emf (full line) and the measured back-emf (dots). The most notable effect of the ripple is a variation in the back-emf amplitude of $12 \%$, which would not be present in the simulated back-emf when the speed ripple would be neglected. As mentioned before, neglecting this impact in an application where torque ripple must be minimized, would not result in a constant torque since the back-emf differs from the constant-speed back-emf.

\section{B. Measurement under load}

A resistive load of $50 \Omega$ is connected to the PMSM in wye without neutral connection, resulting in an electric output power of $480 \mathrm{~W}$. The back-emf can no longer be measured directly. Therefore, the terminal voltage $V$ and current $I$ are measured instead. Fig. 12 shows the result of this measurement.

The speed has an average value $\Omega_{0}$ of $172 \mathrm{rpm}$ and several harmonics, again resulting in a 5\% variation of the speed. The measured speed (dots) was filtered in the frequency domain. The first 10 components where retained, resulting in a filtered speed waveform (full line). Both the voltage $V$ and current $I$ show a $10 \%$ variation of the amplitude due to the speed ripple. This variation can also be expected in the back-emf.

Although the back-emf waveform can not be measured directly, it can be estimated in two ways. On one hand, the back-emf can be calculated from the measured speed $\Omega$ and the model developed in this paper. The resulting waveform is shown as a full line in Fig. 12. On the other hand, the back-emf can be calculated from the measured $V$ and $I$ by estimating the voltage drop over the machine impedance:

$$
E \simeq V+R_{s} I+L_{s} \frac{d I}{d t}
$$



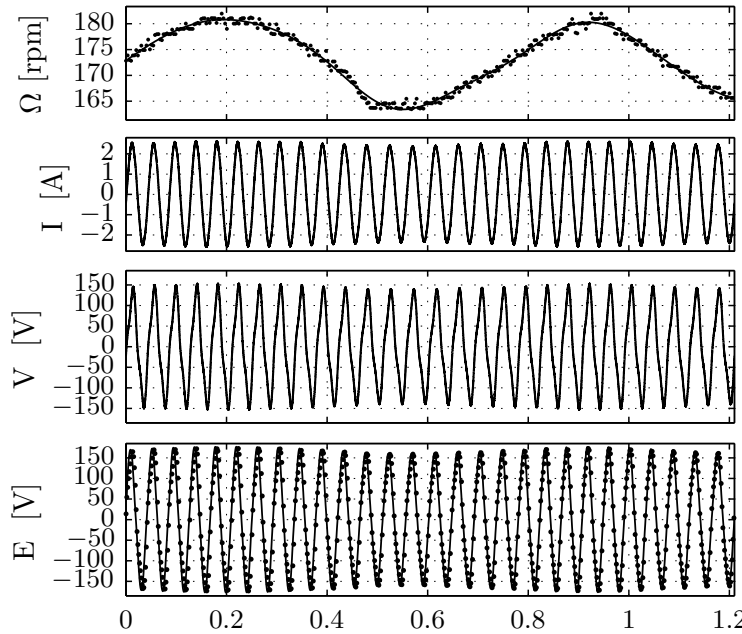

Fig. 12. Measurement under load: $\Omega(t), I(t), V(t)$ and calculated $E(t)$

The result of this calculation is shown with dots in Fig. 12 There is a clear correspondence between both back-emf waveforms. As expected, both have an amplitude variation of $10 \%$. Again, this variation would not be present if the speed ripple was neglected.

\section{CONCLUSIONS}

The effect of speed ripples on the flux and back-emf waveform was discussed in detail. Although the existence of this effect was intuitively clear beforehand, no mathematical model was found in literature to calculate the additional harmonics caused by speed ripples. Therefore, in this paper, the effect was modeled mathematically and a simplified analytical expression for the harmonics was found. The simplified model has the advantage that the back-emf can be seen as the superposition of the classical constant-speed back-emf $E_{0}$ and an additional term $E_{v}$ determined by the speed ripples. The simplified model allows to simulate the back-emf waveform in the presence of speed ripples, which can be of high importance in vibrationsensitive applications.

The model was verified in two situations, i.e., an imposed speed ripple and a speed ripple caused by a torque ripple. This has shown that the accuracy of the simplified model was sufficient, even for extreme ripple amplitudes. Finally, two measurements have been performed on a test-setup. First, a no-load test has been performed in which the back-emf could be measured directly, showing the impact of the speed ripple. A correspondence between the simulated and measured backemf was shown. Second, a load was connected to the machine terminals. From the measured terminal voltage, current and speed, the back-emf could be reconstructed, again showing the impact of the speed ripple and a correspondence of the model.

\section{ACKNOWLEDGMENT}

The research of Jeroen D. M. De Kooning is funded by a Ph.D. fellowship from the Special Research Fund (BOF) of Ghent University (Belgium). The work of Tine Vandoorn is supported by a Ph.D. fellowship from FWO-Vlaanderen (Research Foundation - Flanders, Belgium).

\section{REFERENCES}

[1] H. Huang, C. Mao, J. Lu and D. Wang, "Small-signal modelling and analysis of wind turbine with direct drive permanent magnet synchronous generator connected to power grid," IET Renewable Power Generation, vol. 6, pp. 48-58, Jan. 2012.

[2] H. Vansompel, P. Sergeant, L. Dupre and A. Van den Bossche, "A combined wye-delta connection to increase the performance of axialflux pm machines with concentrated windings," IEEE Trans. on Energy Conversion, vol. 27, pp. 403-410, June 2012.

[3] C. C. Hwang, P. L. Li, C. T. Liu and C. Chen, "Design and analysis of a brushless DC motor for applications in robotics," IET Electric Power Applications, vol. 6, pp. 385-389, Aug. 2012.

[4] Joon-Ho Lee, Dong-Hun Kim and Il-Han Park, "Minimization of higher back-emf harmonics in permanent magnet motor using shape design sensitivity with b-splice parameterization," IEEE Trans. on Magnetics, vol. 39, pp. 1269-1272, May 2003.

[5] F. Marignetti, V. Delli Colli, R. Di Stefano and A; Cavagnino, "Design issues of a fractional-slot windings axial flux pm machine with soft magnetic compound stator,' in Proceedings of the 33rd Annual Conference of the IEEE Industrial Electronics Society (IECON2007), Nov. 5 - 8, 2007.

[6] J. Y. Chai and C. M. Liaw, "Reduction of speed ripple and vibration for switched reluctance motor drive via intelligent current profiling," IET Electric Power Applications, vol. 4, pp. 380-396, May 2010.

[7] J. Sopanen, V. Ruuskanen, J. Nerg and J. Pyrhonen, "Dynamic torque analysis of a wind turbine drive train including a direct-driven permanent magnet generator," IEEE Trans. on Industrial Electronics, vol. 58, pp. 3859-3867, Sept. 2011.

[8] D. S. L. Dolan and P. W. Lehn, "Simulation model of wind turbine 3p torque oscillations due to wind shear and tower shadow," IEEE Trans. on Energy Conversion, vol. 21, pp. 717-724, Sept. 2006.

[9] L. B. Ristic and B. I. Jeftenic, "Implementation of fuzzy control to improve energy efficiency of variable speed bulk material transportation," IEEE Trans. on Industrial Electronics, vol. 59, no. 7, 2012.

[10] T.-L. Chern, L.-H. Liu, P.-L. Pan, T.-M. Huang, D.-M. Tsay, J.-H. Kuang and L.-J. Chen, "Digital signal processing-based sensor-less permanent magnet synchronous motor driver with quasi-sine pulse-width modulation for air-conditioner rotary compressor," IET Electric Power Applications, vol. 6, pp. 302-309, July 2012.

[11] Y. Y. Xia, J. E. Fletcher, S. J. Finney, K. H. Ahmed and B. W. Williams, "Torque ripple analysis and reduction for wind energy conversion systems using uncontrolled rectifier and boost converter," IET Renewable Power Generation, vol. 5, pp. 377-386, Sept. 2011.

[12] M. J. Jin, Y. Wang, J. X. Shen, P. C. K. Luk, W. Z. Fei and C. F. Wang, "Cogging torque suppression in a permanent-magnet fluxswitching integrated-starter-generator," IET Electric Power Applications, vol. 4, pp. 647-656, Sept. 2010.

[13] S. S. Bharatkar, R. Yanamshetti, D. Chatterjee and A. K. Ganguli, "Dualmode switching technique for reduction of commutation torque ripple of brushless dc motor," IET Electric Power Applications, vol. 5, pp. 193 202, Jan. 2011.

[14] J. Y. Hung and Z. Ding, "Design of currents to reduce torque ripple in brushless permanent magnet motors," IEE Proceedings B, vol. 140 , pp. 260-266, July 1993.

[15] A. A. Abd Hafez, R. Todd, A. J. Forsyth and A. M. Cross, "Direct current ripple compensation for multi-phase fault-tolerant machines," IET Electric Power Applications, vol. 5, pp. 28-36, Jan. 2011.

[16] J. X. Shen, Z. Q. Zhu and D. Howe, "Sensorless flux-weakening control of permanent magnet brushless machines using third harmonic back EMF," IEEE Trans. on Industry Applications, vol. 40, pp. 1629-1636, Nov. 2004

[17] M. Njeh, S. Cauet, P. Coirault and P. Martin, " $\mathrm{H}_{\infty}$ control strategy of motor torque ripple in hybrid electric vehicles: an experimental study," IET Control Theory \& Applications, vol. 5, pp. 131-144, Jan. 2011.

[18] D.-H. Lee and J.-W. Ahn, "A current ripple reduction of a high-speed miniature brushless direct current motor using instantaneous voltage control," IET Electric Power Applications, vol. 3, pp. 85-92, Mar. 2009.

[19] H. Zhu, X. Xiao and Y. Li, "Torque ripple reduction of the torque predictive control scheme for permanent-magnet synchronous motors," IEEE Trans. on Industrial Electronics, vol. 59, pp. 871-877, Feb. 2012.

[20] M. C. Paicu, I. Boldea, G. D. Adreescu and F. Blaabjerg, "Very low speed performance of active flux based sensorless control: interior permanent magnet synchronous motor vector control versus direct torque and flux control," IET Electric Power Applications, vol. 3, pp. 551-561, Nov. 2009. 